\title{
Variabilidad fenotípica del déficit de GLUT1: ¿cuándo es necesario sospechar?
}

\section{Phenotypic variability of GLUT1 deficiency: when is necessary to suspect?}

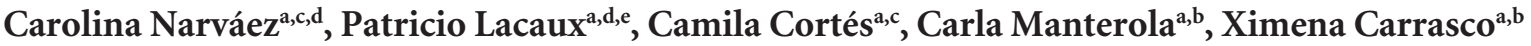 \\ aFacultad de Medicina, Universidad de Chile. Hospital Dr. Luis Calvo Mackenna. Chile \\ ${ }^{b}$ Clínica Alemana de Santiago. Chile \\ 'Programa Formación de Especialistas Neurología Pediátrica, Facultad de Medicina Universidad de Chile \\ dHospital San Juan de Dios, Chile \\ eClínica Santa María, Chile
}

Recibido: 15 de abril de 2019; Aceptado: 22 de septiembre de 2019

\section{¿Qué se sabe del tema que trata este estudio?}

Esta patologia trata de una combinación clínica variable de: epilepsia precoz y refractaria (típicamente generalizada, incluyendo ausencias), afectación cognitiva (desde dificultades atencionales a déficit intelectual severo), $y$ trastornos del movimiento (desde distonía o corea paroxísticos, hasta ataxia y espasticidad persitentes y severas). Su diagnóstico y tratamiento son altamente específicos, y la sospecha clínica resulta fundamental.

\begin{abstract}
¿Qué aporta este estudio a lo ya conocido?
El caso de dos medio-hermanos que presentamos enfatiza que, tras una epilepsia de ausencias iniciada antes de los 4 años, se debe sospechar este cuadro, más aún si se suma un trastorno motor paroxístico. Aunque no es lo habitual, demuestra además que existen casos familiares, de herencia autosómica dominante. A partir de ellos, aportamos una revisión actualizada del tema.
\end{abstract}

\section{Resumen}

La deficiencia del transportador de glucosa tipo 1 constituye un síndrome (SD-GLUT1), provocado por la mutación del gen SLC2A1, que codifica la proteína transportadora de glucosa al encéfalo. Las manifestaciones neurológicas se dan en tres dominios principales: crisis epilépticas, movimientos anormales y alteraciones cognitivas. El diagnóstico se presume ante el hallazgo de hipoglucorraquia y se confirma mediante el análisis molecular del gen. La importancia de precisarlo radica en que tiene tratamiento específico, la dieta cetogénica. Objetivo: Analizar dos casos clínicos de SD-GLUT1 de presentación atípica, destacando la variabilidad del fenotipo. Caso Clínico: Presentamos el caso de dos hermanos cuyas manifestaciones fueron crisis epilépticas de tipo ausencias típicas, y un trastorno paroxístico del movimiento. Los pacientes fueron estudiados encontrándose hipoglucorraquia en ambos y se confirmó diagnóstico de SD-GLUT1 con estudio molecular. El tratamiento específico con dieta cetogénica logró buena respuesta. Conclusiones: Exponemos sus características clínicas peculiares que nos permitieron sospechar este cuadro, de espectro fenotípico amplio, cuyo diagnóstico y tratamiento, correcto y oportuno, puede mejorar significativamente la calidad de vida de los afectados.
Palabras clave:

Transportador de glucosa; Epilepsia refractaria; Movimientos anormales; Discapacidad intelectual; Dieta cetogénica 


\begin{abstract}
Glucose Transporter Type 1 Deficiency Syndrome (GLUT1-DS) is caused by the SLC2A1 gene mutation, which encodes the glucose transporter proteins to the brain Neurological manifestations occur in three main domains: seizures, abnormal movements, and cognitive disorders. The diagnosis is presumed upon the finding of low CSF glucose and confirmed by the gene molecular analysis. Accurate diagnosis is important because it has a specific treatment, which is ketogenic diet. Objective: To analyze two SD-GLUT1 pediatric patients with unusual phenotype. Clinical Case: We present the case of two siblings who presented absence seizures and a paroxysmal movement disorder. Both patients were studied, finding low CSF glucose. The diagnosis of GLUT1-DS was confirmed with molecular analysis. Specific treatment with ketogenic diet achieved good response in both cases. Conclusions: We present their peculiar clinical characteristics that allowed us to suspect this wide phenotypic spectrum. Correct and timely diagnosis and treatment can significantly improve the quality of life of those affected.
\end{abstract}

\section{Keywords:}

Glucose transporter; Refractory epilepsy; Abnormal movements; Intellectual disability; Ketogenic diet

\section{Introducción}

La glucosa es la principal fuente de energía del Sistema Nervioso en general y del cerebro en particular. El paso de ésta a través de la barrera hematoencefálica (BHE) es dependiente de la proteína transportadora de glucosa, GLUT1 ${ }^{1}$. Esta proteína de membrana es codificada por el gen SLC2A1, cuyas mutaciones causan el SD-GLUT12 ${ }^{2}$. La enfermedad fue descrita por primera vez en 1990 por De Vivo, quien reportó dos pacientes con epilepsia de difícil manejo en el período de lactante y retraso del desarrollo psicomotor, asociada a hipoglucorraquia persistente, sugerente de una falla en el transporte de glucosa por la BHE. Tras la descripción inicial, el fenotipo se ha ampliado, reportándose diversas manifestaciones clínicas con severidad variable ${ }^{3}$. El objetivo general del presente artículo es comunicar una forma atípica de presentación de SD-GLUT1, considerando que es un cuadro con un fenotipo variable. Exponemos dos casos con sintomatología relativamente leve que podrían pasar inadvertidos si se desconocen las variantes posibles del cuadro.

\section{Caso Clínico}

\section{Caso 1}

Paciente de sexo femenino, primera hija de padres sanos no-consanguíneos, sin antecedentes médicos relevantes conocidos. Desde los 3 años de edad presenta eventos paroxísticos en vigilia, caracterizados por desconexión del medio y mirada fija, durante unos 30 segundos, cuya frecuencia fue progresiva, llegando hasta 15 episodios diarios. Fue evaluada por Neuropediatra a los 4 años, encontrándose examen neurológico y desarrollo psicomotor normales. El electroencefalograma mostró actividad de base normal, con paroxismos de actividad epileptiforme interictal tipo espiga onda generalizada. La resonancia magnética cerebral resultó normal. Se diagnosticó Epilepsia de ausencias infantil e inició tratamiento con ácido valproico, con buena respuesta, sin nuevos eventos por 2 años. A los 6 años, tras seis meses de suspendido el fármaco antiepiléptico, inició episodios de agitación motora de las extremidades inferiores, asociadas a dolor e incomodidad, que impedían la bipedestación. Los eventos se presentaban en vigilia, sin compromiso de conciencia, con una duración aproximada de 1 minuto y una frecuencia de 1 a 2 al mes, sin un factor desencadenante claro, aunque muchos de ellos sucedían previos a dormir. Se planteó y descartó, en primera instancia, un síndrome de piernas inquietas. Luego de 4 años sin crisis epilépticas, presentó nuevamente crisis de ausencias. Se reinició tratamiento con ácido valproico, al que se le agregó posterioremnte clobazam por falta de respuesta. Adicionalmente se lograron objetivar con video doméstico las crisis de movimientos anormales de las piernas, que se catalogaron como corea paroxístico. El examen neurológico y rendimiento escolar se mantenían normales.

\section{Caso 2}

Paciente de sexo masculino, hermano menor por línea materna de Caso 1. A los 3 años fue evaluado por episodios de caídas abruptas, con aparente pérdida del tono y fuerza de extremidades inferiores, sin compromiso de conciencia. Los episodios duraban aproximadamente 5 min y tenían una frecuencia de 1 a 2 por mes. No se identificó ningún factor gatillante. Se sospechó y descartó una parálisis periódica hiperkalémica. A la edad de 4 años se agregaron crisis epilépticas de ausencias típicas, con un electroencefalograma similar al del Caso 1. Las crisis persistieron tras la indicación de ácido valproico. El examen neurológico era normal, salvo leve hiperactividad e impulsividad. 
Se sospechó en consecuencia un SD-GLUT1 autosómico-dominante. El estudio de líquido cefalorraquídeo (LCR) de ambos hermanos, mostró hipoglucorraquia. (Caso 1: glucorraquia $33 \mathrm{mg} / \mathrm{dl}$, relación glucorraquia/glicemia: 0,39 . Caso 2: glucorraquia $31 \mathrm{mg} /$ dl, relación glucorraquia/glicemia: 0,55 [valor normal relación glucosa LCR/sangre es $>0,4]$ ). El diagnóstico se confirmó con estudio de secuenciación del gen SLC2A1 en el Caso 1, que demostró una mutación puntual heterocigota (c.694C $>$ T - p.Arg32Cys). Se les indicó a ambos hermanos iniciar dieta cetogénica. Se mantuvo el tratamiento antiepiléptico, el cual no requirió modificación, logrando remisión de las crisis epilépticas y de las diskinesias de extremidades inferiores a los 2 meses de su inicio. Hasta la fecha se mantienen asintomáticos con un seguimiento de 18 meses. Desde el punto de vista cognitivo, no se observó mayor cambio clínico luego del inicio de la dieta cetogénica. Sin embargo, no se contó con evaluaciones psicométricas.

\section{Discusión}

El cerebro es un órgano altamente requirente de energía, sin embargo posee escasa capacidad de almacenamiento energético como glucógeno, necesitando un suministro continuo de glucosa o, en su defecto, el aporte de un combustible alternativo como los cuerpos cetónicos ${ }^{3}$. GLUT1 es una glicoproteína unida a membrana expresada en eritrocitos, endotelio capilar cerebral y astrocitos ${ }^{4}$, que permite el paso de glucosa por la BHE.

SLC2A1 es el gen que codifica GLUT1, se localiza en el cromosoma 1 (1p34.2), tiene $35 \mathrm{~kb}$ y consta de 10 exones $^{5}$. Diversas mutaciones en este gen (cerca de 100 descritas a la fecha), producen una pérdida de fun-

\footnotetext{
Tabla 1. Características de las crisis de ausencias que hacen sospechar GLUT1-SD

Inicio precoz $<4$ años

$>$ Presentes temprano en la mañana o en ayuno

$>$ Mejoran con la ingesta calórica

$>$ Farmacorresistencia

> Otro tipo de crisis asociadas precozmente (mioclonías, tónico-clónico y de forma menos frecuente focal o tónicas)

$>$ EEG con actividad interictal breve y frecuente

> Descargas generalizadas irregulares en forma y frecuencia

$>$ Historia de otro tipo de manifestación neurológica (hipotonía, microcefalia, ataxia, migraña, inestabilidad, movimientos involuntarios paroxísticos)

Dificultades cognitivas y atencionales pueden emerger posteriormente
}

ción, es decir, reducen el aporte de glucosa al encéfalo, con la consecuente deficiencia energética que resulta deletérea para la función cerebral y el neurodesarrollo ${ }^{3}$. Se ha descrito que el $84 \%$ de las alteraciones genéticas corresponden a mutaciones puntuales y el $13 \%$ a deleciones/duplicaciones ${ }^{2}$. Modelos animales, estudios electrofisiológicos y de neuroimágenes funcionales con glucosa marcada han permitido observar que mutaciones en SLC2A1 producen una disfunción de la conectividad tálamo-cortical, lo que determinaa el frecuente hallazgo de descargas epileptiformes de espiga-onda a $3 \mathrm{~Hz}$ y una sobrecaptación relativa de glucosa en los núcleos basales, que podría relacionarse con los trastornos del movimiento ${ }^{6}$. La mayoría de los pacientes tiene mutaciones de novo heterocigotas. Las formas familiares son generalmente autosómicas dominantes ${ }^{3}$. Las mutaciones bialélicas de SLC2A1 serían letales en la mayoría de los casos, aunque se han reportado casos con transmisión autosómica recesiva ${ }^{7,8}$. No existe una correlación definida genotipo-fenotipo, existiendo una alta variabilidad fenotípica, incluso dentro de una misma familia. Pueden influir en el fenotipo otros factores genéticos, epigenéticos o ambientales, así como también la presencia de fuentes alternativas de sustratos naturales para el cerebro, como cuerpos cetónicos ${ }^{3}$.

El espectro de severidad del SD-GLUT1 es amplio, variando desde pacientes con sintomatología mínima y ausencia de clínica entre episodios, hasta pacientes con déficits neurológicos severos y fijos. Las manifestaciones neurológicas priman en este cuadro, identificándose tres tipos de síntomas: crisis epilépticas, trastornos del movimiento y problemas cognitivo-conductuales. El fenotipo del SD-GLUT1 clásico se caracteriza por síntomas persistentes en los 3 dominios. En contraste, los pacientes con fenotipos más leves pueden experimentar síntomas en sólo 1 o 2 dominios, pudiendo presentarse en forma intermitente o persistente 9 . El fenotipo clásico se caracteriza por una epilepsia fármaco-resistente que comienza usualmente en los primeros meses de vida, retraso del desarrollo psicomotor global, microcefalia adquirida, espasticidad, ataxia y movimientos anormales ${ }^{3,5}$. Las crisis epilépticas generalmente se presentan en el periodo de lactantes y son refractarias al tratamiento con fármacos antiepilépticos ${ }^{10,11}$. Se describen varios tipos de crisis epilépticas, siendo las más frecuentes tónicoclónico-generalizadas y ausencias ${ }^{12}$. Algunas claves que deben hacer sospechar el diagnóstico de SD-GLUT1 frente a crisis de ausencia se describen en la tabla 1. Los trastornos del movimiento se observan comúnmente. Los pacientes experimentan una variedad de síntomas motores persistentes y/o paroxísticos ${ }^{9}$, siendo los principalmente descritos: marcha atáxica con o sin espasticidad, distonía, corea, temblor de intención, dispraxia y mioclonías. Desencadenantes comunes de los sínto- 
mas episódicos son el ayuno y el ejercicio, aunque a veces no se puede identificar ningún gatillante, como en los casos reportados en este artículo. Dentro de los eventos paroxísticos que se presentan en SD-GLUT1, cabe destacar la "diskinesia paroxística inducida por ejercicio", caracterizada por episodios de movimientos involuntarios diversos, generalmente de 5 a $30 \mathrm{~min}$ de duración, que se desencadenan por el ejercicio sostenido y suelen comenzar durante la niñez. La distonía de miembros inferiores precipitada por marcha sostenida, o por correr, es la manifestación más común, pero también pueden producirse mioclonías, atetosis, corea y balismo, solos o en combinación ${ }^{9}$. La alta tasa de sospecha clínica y la mayor disponibilidad de estudio genético han permitido ampliar el espectro clínico, encontrándose pacientes sin crisis epilépticas, en quienes los trastornos del movimiento pueden ser la característica más prominente ${ }^{9}$.

La mayoría de los pacientes experimentan algún grado de compromiso cognitivo, que va desde leve dificultad en el aprendizaje a discapacidad intelectual severa. Sin embargo, es importante destacar que existen pacientes con cognición normal ${ }^{9}$. La atención sostenida parece ser una función particularmente afectada en personas portadoras de SD-GLUT1 ${ }^{9}$. Manifestaciones extraneurológicas infrecuentes son anemia hemolítica (atribuible a que GLUT1 es el transportador principal de glucosa en los glóbulos rojos), hepato-esplenomegalia y cataratas ${ }^{3,9}$.

Tras la sospecha clínica, la confirmación diagnóstica se basa en el análisis citoquímico de LCR, que muestra hipoglucorraquia, y el estudio molecular del gen SLC2A1, que demuestra alguna mutación patogénica. Para determinar hipoglucorraquia, es necesario analizar LCR tras cuatro a seis horas de ayuno y obtener una glicemia concomitante normal.

Todos los individuos reportados hasta la fecha han tenido valores de glucosa en LCR $<60 \mathrm{mg} / \mathrm{dL}$ (rango: 16,2 a $52 \mathrm{mg} / \mathrm{dL}$ ), siendo en más del $90 \%$ de los casos $<40 \mathrm{mg} / \mathrm{dL}$, considerado altamente sugerente de SDGLUT1. Por otro lado, los valores entre 40 a 60 podrían tener importancia. Otro valor a analizar es la relación glucosa LCR/glucosa sangre, que suele ser $<0,4$, este valor es menos fiable que el valor absoluto de la glucosa en LCR. La concentración de lactato en LCR es normal-bajo o bajo, a menudo $<11,7 \mathrm{mg} / \mathrm{dl}$ (intervalo de 5,4 a 13,5 mg/dl $)^{2-13}$. SLC2A1 es el único gen hasta ahora conocido como responsable del SD-GLUT1. Se recomienda que el análisis de secuenciación sea realizado primero $y$, si no se identifica ninguna variante patogénica mediante este método, practicar el análisis de deleción/duplicación ${ }^{2}$.

La dieta cetogénica es actualmente la piedra angular del tratamiento ${ }^{3}$, la que fue efectiva en los dos casos en relación al control de las crisis epilépticas y del trastorno del movimiento. El uso de la dieta cetogénica se fundamenta en que cuando el suministro de glucosa es insuficiente, los cuerpos cetónicos son la única fuente de combustible alternativa relevante para el metabolismo cerebral ${ }^{3}$. Debido a la gran demanda de energía durante el desarrollo cerebral, la dieta cetogénica debe iniciarse lo antes posible y ser mantenida al menos hasta la adolescencia, aunque parece ser beneficiosa incluso cuando se inicia en etapas tardías de la niñez ${ }^{18}$. Si existe una baja adherencia, durante la adolescencia se podría cambiar a la dieta cetogénica modificada de Atkins, la que es menos restrictiva. Dentro de los resultados usuales obtenidos con la dieta cetogénica se incluyen remisión total, o al menos reducción, de las crisis epilépticas, rápida desaparición de los trastornos paroxísticos no epilépticos, lenta reducción de los trastornos motores persistentes, pero escasa mejoría en aspectos del neurodesarrollo y las funciones cognitivas ${ }^{3}$.

Adicionalmente se pueden usar fármacos para el manejo de síntomas residuales, pero habitualmente éstos no son muy efectivos ${ }^{12,14}$. Acetazolamida puede ser la primera opción para tratar los trastornos del movimiento paroxísticos ${ }^{15,16}$. Se deben evitar fármacos que potencialmente alteran la función de GLUT1, entre los que destaca: cafeína, fenobarbital, diazepam, valproato $y$ antidepresivos tricíclicos ${ }^{14,17}$. Resulta fundamental una apropiada rehabilitación, incluyendo fisioterapia, terapia fonoaudiológica y ocupacional, junto con promover la integración escolar y profesional ${ }^{3}$.

\section{Conclusiones}

El SD-GLUT1 es una enfermedad infrecuente, pero con un creciente número de casos diagnosticados en los últimos años, lo que ha permitido reconocer una amplia variabilidad fenotípica. En este artículo se describen dos pacientes con epilepsia de ausencias de inicio precoz, con buena respuesta inicial a fármacos antiepilépticos, que posteriormente iniciaron movimientos anormales, sin evidencia clínica de compromiso cognitivo. Si bien en el Caso 2 se describe hiperactividad e impulsividad, no es posible determinar si esto se debe a sintomatología relacionada con SD-GLUT1 o a un trastorno de déficit de atención e hiperactividad altamente prevalente en la población. Ambos casos presentaron una excelente respuesta al tratamiento con dieta cetogénica. El conocimiento y un alto índice de sospecha diagnóstica permite diagnosticar pacientes con fenotipos variables. Actualmente se dispone del estudio genético molecular, permitiendo realizar el diagnóstico definitivo. La implementación del tratamiento específico y efectivo permite mejorar la calidad de vida de los pacientes. 


\section{Responsabilidades Éticas}

Protección de personas y animales: Los autores declaran que los procedimientos seguidos se conformaron a las normas éticas del comité de experimentación humana responsable y de acuerdo con la Asociación Médica Mundial y la Declaración de Helsinki.

Confidencialidad de los datos: Los autores declaran que han seguido los protocolos de su centro de trabajo sobre la publicación de datos de pacientes.
Derecho a la privacidad y consentimiento informado: Los autores han obtenido el consentimiento informado de los pacientes y/o sujetos referidos en el artículo. Este documento obra en poder del autor de correspondencia.

\section{Conflicto de intereses}

Los autores declaran no tener conflicto de intereses.

\section{Referencias}

1. Ramm-Pettersen A, Nakken K, Haavardsholma K, Selmer K. GLUT1deficiency syndrome: Report of a fourgeneration Norwegian family with a mild phenotype. Epilepsy Behav. 2017;70:1-4.

2. Wang D, Pascual J, De Vivo D. Glucose Transporter Type 1 Deficiency Syndrome. GeneReviews ${ }^{\circledR}$. Disponible en https:// www.ncbi.nlm.nih.gov/books/NBK1430/ (último acceso el 20 de septiembre de 2019).

3. Gras D, Roze E, Caillet S, et al. GLUT1 deficiency syndrome: An update. Rev Neurol (Paris). 2014;170:91-9.

4. Vannucci S, Gibbs E, Simpson I. Glucose utilization and glucose transporter proteins GLUT1 and GLUT3 in brains of diabetic $(\mathrm{db} / \mathrm{db})$ mice. Am J Physiol. 1997;272:267-74.

5. De Giorgis V, Veggiotti P. GLUT1 deficiency syndrome 2013: Current state of the art. Seizure. 2013;22:803-11.

6. Pascual JM, Ronen GM. Glucose transporter type I deficiency (G1D) at 25 (1990-2015): Presumtions, facts and the lives of persons with this rare disease.
Pediatr Neurol. 2015;53(5):379-93.

7. Klepper J, Scheffer H, Elsaid M, Kamsteeg E, Leferink M, Ben-Omran T. Autosomal recessive inheritance of GLUT1 deficiency syndrome. Neuropediatrics. 2009;40(5):207-10.

8. Rotstein M, Engelstad K, Yang H, et al. GLUT1 deficiency: inheritance pattern determined by haploinsufficiency. Ann Neurol. 2010;68(6):955-8.

9. Pearson T, Akman C, Hinton V, Engelstad K, De Vivo D. Phenotypic Spectrum of Glucose Transporter Type 1 Deficiency Syndrome (Glut1 DS). Curr Neurol Neurosci Rep. 2013;13(4):342.

10. De Vivo DC, Leary L, Wang D. Glucose transporter 1 deficiency syndrome and other glycolytic defects. J Child Neurol. 2002;17 Suppl 3:3S15-23, discussion 3S24-5.

11. Leary L, Wang D, Nordli Jr D, Engelstad K, De Vivo D. Seizure characterization and electroencephalographic features in Glut-1 deficiency syndrome. Epilepsia. 2003;44(5):701-7.

12. Pong A, Geary B, Engelstad K, Natarajan A, Yang H, De Vivo DC. Glucose transporter type I deficiency syndrome: epilepsy phenotypes and outcomes. Epilepsia. 2012;53(9):1503-10.

13. Campistol J. Epilepsy in Inborn Errors of Metabolism with Therapeutic Option. Seminars in Pediatric Neurology. 2016;23(4):321-31.

14. Cano A, Ticus I, Chabrol B. Glucose transporter type 1 (GLUT1) deficiency. Rev Neurol. 2008;164(11):896-901.

15. Anheim M, Maillart E, VuillaumierBarrot S, et al. Excellent response to acetazolamide in a case of paroxysmal dyskinesias due to GLUT1 deficiency. J Neurol. 2011;258(2):316-7.

16. Chambon $R$, Vuillaumier-Barrot $S$, Seta N, Wagner S, Sarret C. Partial effectiveness of acetazolamide in a mild form of GLUT1 deficiency: a pediatric observation. Mov Disord. 2013;28(12):1749-51.

17. Brockmann $\mathrm{K}$. The expanding phenotype of GLUT1-deficiency syndrome. Brain Dev. 2009;31(7):545-52.

18. Gramer G, Wolf N, Vater D, et al. Glucose transporter-1 (GLUT1) deficiency syndrome: diagnosis and treatment in late childhood. Neuropediatrics. 2012; 43(3): 168-71. 